REVISTA DE GEOCIÊNCIAS DO NORDESTE

Northeast Geosciences Journal

v. $6, n^{\circ} 2$ (2020)

ISSN: 2447-3359

https://doi.org/10.21680/2447-3359.2020v6n2ID19280

\title{
ÍNDICE DE VULNERABILIDADE SOCIAL À DESASTRES NATURAIS PARA ÁREAS URBANAS
}

\author{
Lindberg Nascimento Júnior ${ }^{1}$; João Lima \\ Sant'Anna Neto ${ }^{2}$
}

${ }^{1}$ Doutor em Geografia, Departamento de Geociências, Universidade Federal de Santa Catarina (UFSC), Florianópolis/SC, Brasil.

ORCID: https://orcid.org/0000-0003-0276-2933

Email: lindberg.junior@ufsc.br

${ }^{2}$ Doutor em Geografia, Departamento de Geografia, Universidade Estadual Paulista (Unesp), Presidente Prudente/SP, Brasil.

ORCID: https://orcid.org/0000-0002-5947-1734

Email: joaolima@fct.unesp.br

\section{Resumo}

Apresenta-se uma aplicação do Social Vulnerability Index SoVI ${ }^{\circledR}$, com o interesse de contribuir aos estudos geográficos da vulnerabilidade social à desastres naturais em áreas urbanas. Para exemplificação utilizou-se dados do censo demográfico por setores censitários urbanos da cidade de Santos, na Zona Costeira Paulista. Foram detectadas nove componentes que explicam $75,6 \%$ da variância da vulnerabilidade em Santos. A primeira componente é a desigualdade social, racial e gênero, uma vez que, as jovens, alfabetizadas, portadoras da cor parda e residentes em domicílios improvisados sem rendimento, são o grupo populacional mais vulnerável. Valores altos de SoVI ${ }^{\circledR}$ foram observados nos setores norte e noroeste da cidade, na zona portuária, no centro, e nas áreas de aglomerados subnormais. Os menores índices estão distribuídos, no setor sul, especialmente nas proximidades da orla praiana. Os resultados, indicam que em Santos, a espacialização dos índices apresenta-se como um subproduto de eventos históricos e contextos sócioespaciais, que explicam como os processos de urbanização (precária e segregada) incorporaram diferentes níveis de vulnerabilidade social, com privações de direitos sociais, à cidade e à justiça social. Nestes aspectos o SoVI ${ }^{\circledR}$ é estratégia interessante para o estudo dos riscos e desastres naturais, desde que os processos metodológicos de elaboração sejam claros e substanciados teoricamente.

Palavras-chave: Cartografia dos riscos; Vulnerabilidade; SoVI ${ }^{\circledR}$.

\section{SOCIAL VULNERABILITY INDEX TO NATURAL DISASTERS FOR URBANS AREAS}

\begin{abstract}
An application of Social Vulnerability Index - SoVI® is presented, with the interest of contributing to vulnerability to natural disasters in urban areas on geographic studies. As an example, we used demographic census data by urban census sectors of Santos city - São Paulo Coastal Zone. Nine mean components of social vulnerability in Santos were detected and it explain $75.6 \%$ of variance. The first component is social, racial and gender inequality and the most vulnerable population group is the youngs wemen, browns, without income, literate, and residents in improvised households. High $\mathrm{SoVI}^{\circledR}$ values were observed in northern sectors of city, which comprises the port area, the center and the areas of subnormal households. The lowest indexes are distributed in the southern sector, especially on the beach front. In Santos, the mapping of SoVI ${ }^{\circledR}$ shows an effective subproduct of historical events and socio-spatial contexts, which explains how the processes of precarious and segregated urbanization incorporated different levels of social vulnerability with deprivation of social rights, to thee city and social justice. The use of SoVI ${ }^{\circledR}$ for urban areas can be an interesting strategy for natural risks and disasters studies, provided that the methodological processes of elaboration and organization are theoretically clear and substantiated.
\end{abstract}

Keywords: cartography of risks; vulnerability; SoVI ${ }^{\circledR}$.

\section{ÍNDICE DE VULNERABILIDAD SOCIAL A DESASTRES NATURALES PARA ÁREAS URBANAS Resumen}

Se presenta una aplicación del Índice de Vulnerabilidad Social SoVI ${ }^{\circledR}$, con el interés de contribuir al estudio geográfico de la vulnerabilidad social a los desastres naturales en las zonas urbanas. Como ejemplo, se utilizaron datos del censo demográfico por sectores censales urbanos en la ciudad de Santos - en la Zona Costeira Paulista. Se detectaron nueve componentes que explican el 75.6\% de la varianza de vulnerabilidad en Santos. La primera componente es la desigualdad social, racial y de género, ya que las jóvenes, alfabetizadas, de color marrón y que viven en hogares improvisados sin ingresos, son el grupo de población más vulnerable. Se observaron valores altos de SoVI ${ }^{\circledR}$ 
en los sectores norte y noroeste de la ciudad, el área del puerto, el centro y las áreas de aglomerados subnormales. Los índices más bajos se distribuyen en el sector sur, especialmente en las cercanías de la playa. Los resultados indican que en Santos, la espacialización de los índices se presenta como un subproducto de eventos históricos y contextos socioespaciales que explican cómo los procesos de urbanización (precarios y segregados) incorporaron diferentes niveles de vulnerabilidad social con privaciones de derechos y a la ciudad. En estos aspectos, SoVI ${ }^{\circledR}$ es una estrategia interesante para el estudio de riesgos y desastres, siempre que los procesos de elaboración metodológica sean claros y estén teóricamente fundamentados.

Palabras clave: Cartografía de los riesgos; Vulnerabilidad; SoVI ${ }^{\circledR}$.

\section{INTRODUÇÃO}

Processos de elaboração de índices de vulnerabilidade, que se baseiam em ferramentas estatísticas e geotecnológicas, podem ser considerados como uma das possibilidades mais efetivas para avaliação, controle e gestão dos desastres. De forma abragente, essas métricas podem auxiliar tanto orientações para decisões políticas de defesa e proteção civil, quanto também problematizar as estruturas internas que configuram e organizam os riscos dos lugares (BIRKMANN, 2006; CUTTER, 1996; 2003).

Admitidas como opções metodológicas no estudo do risco, os indices representam medidas gráficas e cartográficas da vulnerabilidade, servindo como parâmetro de conhecimento da espacialidade do risco, como também para análises comparadas do potencial de perda (CUTTER, 1996; 2003), da capacidade de suporte (CUNHA, 2013), e dos níveis de exposição à eventos periosos (DAUPHINÉ, 2005; VEYRET, 2007).

Do conjunto de indices de vulneraiblidade aplicados nos estudos geográficos dos desastres naturais, o modelo Social Vulnerability Index - SoVI ${ }^{\circledR}$ (Índice de Vulnerabilidade Social) desenvolvido por Cutter et al. (2003) é um dos que tem apresentado boa funcionalidade de mapeamento, e também aberturas para discussão teórico-metodológica da análise dos riscos. Para além da importancia na decisão e das formas de aplicação, o SoVI ${ }^{\circledR}$ tem subsidiado interpretações de que as diferentes possibilidades de adtapação, de controle e de surporte a eventos perigosos expressam a estrutura e organização socioespacial dos riscos e da vulnerabilidade do lugar (CUTTER et al, 2003; CUTTER, 2005; 2011).

Das aplicações do SoVI ${ }^{\circledR}$ que foram submetidas no debate acadêmico, os/as autores/as utilizaram recortes territoriais de municípios para escalas nacionais e regionais (CUTTER, 1996; 2003; CUTTER et al, 2003; MENDES et al., 2011; HUMMEL et al., 2016; ARMOND, 2018), e setores censitários para bacias hidrográficas (ALMEIDA, 2012). Nesse trabalho é apresentado a aplicação do SoVI ${ }^{\circledR}$ em areas urbanas, cujo o objetivo é oferecer elementos para um debate geográfico sobre a estrutura, componentes e os processos que organizam a vulnerabilidade social à desastres naturais nas áreas urbanas.

O pressuposto é que, no estudo geográfico dos desastres nas areas urbanas, o SoVI ${ }^{\circledR}$ deve estar subsidiado por uma adaptação metodológica, sem perder o encontro dos contextos e particularidades socioespaciais que definem a vulnerabilidade do lugar (CUTTER, 1996; 2003; CUTTER et al, 2003), conforme exemplificado por Nascimento Jr (2018), Rampazo (2019) e Teobaldo Neto (2019).

Diante do exposto, apresenta-se incialmente uma discussão sobre as caracteristiicas conceituais e estruturais do indice proposto por Cutter et al. (2003), indicando sua relação com os interesses aos estudos geográficos do risco e dos desastres. Em seguida, expoem-se os ajustes e as adaptações do modelo para areas urbanas, indicando a cidade de Santos como universo de análise. Os resultados e a discussão são descritos na terceira parte. Finaliza-se o texto com as considerações finais.

\section{CONCEITO E ESTRUTURA DO SoVI ${ }^{\circledR}$}

O SoVI ${ }^{\circledR}$ é uma avaliação quantitativa das características que influenciam a vulnerabilidade social aos perigos naturais, e facilita a comparação entre unidades geográficas (estado, municípios, cidades, distritos, setores censitários, etc) em termos de valores relativos do potencial de perda (CUTTER, 1996; 2003; 2005; 2011).

O modelo permite estruturar a vulnerabilidade associando uma diversidade variáveis socioeconômicas e demográficas, inserindo dimensões de propensão e de exposição ao risco, considerando finalmente o perfil dos residentes, seus rendimentos, etnia/raça/cor, alfabetização, gênero, estrutura etária, formação, origem, status social, etc, além de parâmetros da qualidade ambiental (coleta de lixo, abastecimento, aborização, iluminação, equipamentos adaptados, etc) e das condições de moradia (tipos de domicílios, infraestruturas, etc) (CUTTER et al, 2003; 2009; CUTTER e FINCH, 2008).

Ususalmente, os perfis populacionais são gerados a partir da informação de censos, em integração com outros bancos de dados (como saúde, mobilidade, transporte, direitos sociais, etc), que submetidos a procedimentos estatísticos específicos, reduzem o número de variáveis a um conjunto menor de fatores e/ou componentes, utilizadas para descrever a organização da vulnerabilidade do lugar (CUTTER et al., 2003).

Desde a primeira aplicação, em Cutter et al. (2003), o SoVI ${ }^{\circledR}$ subsidiou a explicação de como a vulnerabilidade à desastres naturais acontece nos municípios estados-unidenses e se configura regionalmente. Desde então, o indice tem sido utilizado por diversos autores em diferentes contextos: Mendes et al. (2011) em Portugal, Almeida (2012) para a bacia do rio Maranguapinho, na Região Metropolitana de Fortaleza, Hummel et al., (2016) para os municípios brasileiros, e Armond (2018) para os muncípios fluminenses.

Esses/as autores/as não ofereceram paramêtros de similaridade com os resultados encontrados por Cutter et al. (2003). Isso acontece por que são as condições e contextos históricos e scoiespaciais dos lugares que organizam e estruturam vulnerabilidade social, e fazem com que cada universo de análise apresente sempre um resultado particular em seu quadro socioespacial.

Neste sentido, a aplicação do índice tende expandir a problematização dos riscos e dos lugares destacando, na abordagem, o caráter geográfico da vulnerabilidade. Assim, a quantificação em métricas promove tanto qualificação em estudos comparados e de casos, mas também maneiras diferentes, específicas e setorizadas para gestionar os riscos nos lugares. 
Além disso, as aplicações do SoVI ${ }^{\circledR}$ tem marcado uma mudança na formulação geral nos índices de vulnerabilidade que pode ser definida em pelo menos três pontos.

Primeiro, por que o índice enfatiza, teoricamente, que a vulnerabilidade é organizada partir das restrições sociais como base processos de construção dos riscos nos lugares. As desigualdades e a segregação enquanto processos socioespaciais são os principais estruturadores dos riscos e da vulnerabilidade do lugar (CUTTER, 1996; 2003; 2005; 2011). A identificação dos processos socioepaciais colocam em xeque a tradicional perspectiva locacional da vulnerabilidade, que é exclusiva à área exposta ou impactada por algum evento perigoso ou desastre. Em outras palavras, a abordagem politiza a análise do risco, e sugere a superação da lógica reducionista do perigo, que ao invés de naturalizar os processos sociais e a culpabilizar os sujeitos pela sua condição de vulnerabilidade, evidencia sob os quais processos as populações e os lugares estão ou foram submetidas à vulnerabilização (ACSELRAD, 2006; DUTRA, 2015).

A segunda definição problematiza os parâmetros bases para a preparação e resposta aos desastres. Considera-se para isso hierarquização e a combinação entre perfiis populacionais, a partir da estrutura familiar, barreiras linguísticas e educacionais, disponibilidade de recursos, oferta desigual ou limitada de mobilidade por deficiências motoras e locomotivas, acesso à saúde, segurança e proteção, questões relativas à exclusão e formas de violência (pobreza, racismo, machismo, xenofobia, etc), e privação de direitos (civis, humanos, sociais, ambientais, etc).

Nestes aspectos, os limites da vulnerabilidade nos lugares não devem ser orientados e defnidos em função das vítimas do risco e dos perigos, mas fundamentalmente das formas desiguais de proteção e a necessidade de superação (ACSELRAD, 2006). A transformação destes paramêtros exige cada vez mais que ela (a vulnerabilidade) seja entendida de fato como a face invisível dos riscos (CUNHA, 2013), e que tem servido muito mais para a reprodução das relações sociais de produção que organizam e estruram os impactos dos eventos perigosos e dos desastres nos lugares (DUTRA, 2015; NASCIMENTO Jr, 2018; ARMOND, 2018; RAMPAZZO, 2019; TEOBALDO NETO, 2019), do que um bom exercício acadêmico de base cartográfica, que se reduz em um mapa.

A terceira definição orienta-se ao nível teórico, que subjaz o entendimento da vulnerabilidade enquanto medida do risco (VEYRET, 2007). Em outras palavras, o risco só existe e só efetivo quando a vulnerabilidade é conhecida e explicada. Vulnerabilidade não é risco. Mas o risco só existe em função da vulnerabilidade, e só pode ser reduzido (ou até mesmo neutralizado) com a transformação dos processos de vulnerabilização. Em outras palavras, só por mudanças nas estruturas e nas formas de organização da vulnerabilidade do lugar que é possivel gestionar e controlar o risco.

Neste sentido, a pespectiva coloca o $\mathrm{SoVI}^{\circledR}$ como uma ferramenta robusta e sofisticada para representação dessa medida, uma vez que o indíce está aberto metodologicamente à integração de banco de dados de diferentes fontes, e à submissão de testes para controle, ajustes, calibração e validação. Esse ponto é particularmente importante, pois ao passo que a avaliação da vulnerabilidade nos lugares torna-se rigorosa e criteriosa, ela também indica a possibilidade para uma leitura integrada, que deve sugerir transformações dos constextos em que ela (a vulnerabilidade) é produzida (CUTTER, 2011; HUMMEL et al., 2016).

Para contribuir com esta interpretação é importante considerar que Cutter (2011) elege como princípio da análise da vulnerabilidade o reconhecimento geoespacial da investigação com base nos locais, que não deve ser reduzida ao produto cartográfico. Isso por que, os contextos socioespaciais dos lugares, suas diferenciações e contradições internas, devem ser admitidos como parâmetro de análise. O princípio é de que se vulnerabilidade é do lugar, todos os desastres e as respostas são tambem são, e depende de todo o cojunto dos processos em sua espacialidade (CUTTER, 2011).

As três definições enriquecem a questão da vulnerabilidade dentro e fora da geografia, não somente como instrumento de gestão, produto cartográfico e instrumento analítico, mas substancialmente como mecanismo de luta por justiça e direitos sociais (DUTRA, 2015). Esse caráter é relativo à condição de que mesmo antes da ocorrência de um evento perigoso, devem ser admitidas as estruturas socioespacias em sua totalidade, uma vez que são elas organizam as conjunturas dos modelos de desenvolvimento, da marginalização, da segregação socioespacial, das desigualdades sociais, dos sistemas de opressão, dos níveis de pobreza, etc (ALEXANDER, 1995; WHITE et al., 2001; LEONE e VINET; 2006; SMITH, 2001; SILVEIRA, 2014; NASCIMENTO Jr, 2019).

Dialeticamente são esses processos em totalização que submetem as populações e os lugares a situação de emergência permanente (CUTTER, 2006; 2010).

\section{CARACTERIZAÇÃO DO UNIVERSO DE ANÁLISE}

Em vista das possibilidades metodológicas e analíticas que o SoVI ${ }^{\circledR}$ sugere, resta agora oferecer o debate que indique elementos para explicação da vulnerablidade em areas urbanas.

Para isso admitiu-se como unverso de análise a cidade de Santos, cuja área urbana está situada em um ambiente insular, sutado na Zona Costeira Paulista no quadrante $46^{\circ} 24^{\circ}$ e $24^{\circ} 18^{\prime}$ Oeste, e $23^{\circ} 54^{\prime}$ e $24^{\circ} 00^{\prime}$ Sul (Figura 01). A cidade apresenta caracteristicas de clima dominadoss por sistemas tropicais e extratropciais, e pela maritimidade. Essa situação é explicada pela transionalidade climática relativa à localização próxima ao sul do Trópico de Capricórnio.

O sítio é formado por unidades morfoesculturais das Planícies Litorâneas que contemplam pacotes litológicos associados a sedimentos marinhos e fluviais inconsolidados (areias, argilas e cascalhos). Por isso, apresenta altitudes máximas em torno de $20 \mathrm{~m}$ em relação ao nível do mar e declividades muito fracas ou inferiores, em torno de $6 \%$. No setor central, há morros e colinas de gnaisse e de granito que formam uma acentuada alteração de direção norte-sul, com altitudes máximas de 200m acima do nível do mar e as mais altas declividades (ROSS e MOROZ, 1997). 


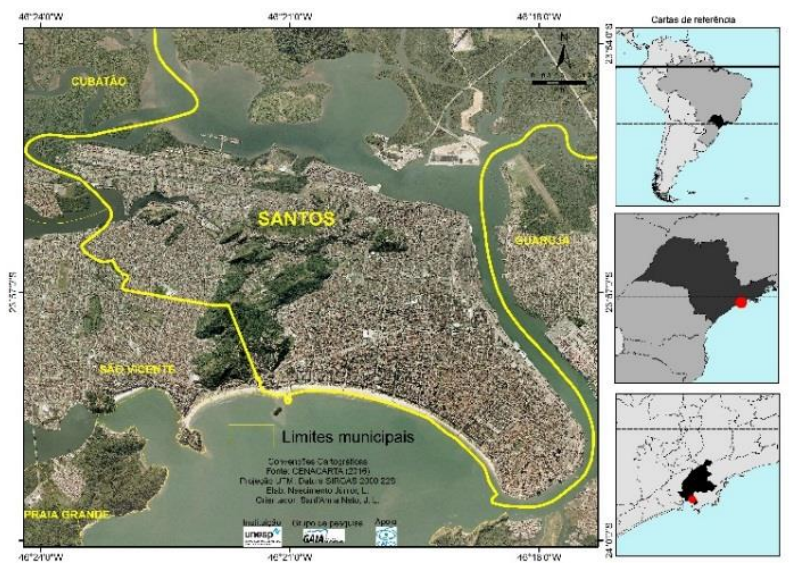

Figura 01 - Localização e situação geográfica da cidade de Santos - SP.

A cidade foi fundada em 1546, sob o modelo de urbanização funcional e segregacionista vinculado à colonização portuguesa, que marcou a cidade inicialmente como unidade geopolítica de controle e domínio territorial (NASCIMENTO Jr, 2018).

Recentemnte o município de Santos foi institucionalizado sede de umas das regiões metropolitanas mais improtantes do Brasil - a Baixada Santista. Por isso, a cidade se apresenta com importante função portuária, industrial e turística, e exerce papel relevante em termos de logística, econômia e cultural na rede urbana em escala regional e nacional.

Atualmente, Santos apresenta incorporação de processos urbanização relevantes para vertifcalização e periferização na estruturação de uma cidade ainda mais segregada do que inicio de sua fundação (MELLO, 2017). Esse contexto é bem evidenciado pela destinação de áreas ambientalmente frágeis e naturalmente suscetíveis e expostas a perigos naturais, que tornaram a cidade um lugar de ocorrência de eventos de diversas gêneses (climáticas, meteorológicas, geomorfológica e oceanográficos) historicamente notificados e anualmente observados.

O resultado é a contrução do espaço urbano santista como um produto-produtor de riscos que tende à cristalização da urbanização desigual e capitalista com incorporação de diversos níveis de segregação socioespacial e vulnerabilidade, e a segmentação de multiplos riscos, dente estes o naturais. Trata-se de um desenvolvimento urbano que não é exclusivo à cidade Santos, por seu exemplo serve como generalização de grande parte das cidades brasileiras e do mundo tropical colonializado, e permite a submissão do SoVI ${ }^{\circledR}$ aplicado às áreas urbanas.

\section{PROCEDIMENTOS METODLÓGICOS}

O SoVI ${ }^{\circledR}$ para a cidade de Santos foi elaborado a partir das informações do Censo Demográfico 2010: Resultados do Universo por setor censitário (IBGE, 2010). Considerou-se as categorias e variáveis desenvolvidas por Cutter et al (2003), Cutter (2005; 2011), Mendes et al. (2011), Almeida (2012), Hummel et al., (2016). O modelo completo e detalhado é apresentado por Nascimento Jr. (2018).

Em síntese, foi incorporado o recorte e a unidade espacial de análise a partir de 654 setores censitários urbanos (Figura 02), com dados de demografia, de domicílios e de entorno dos domicílios.

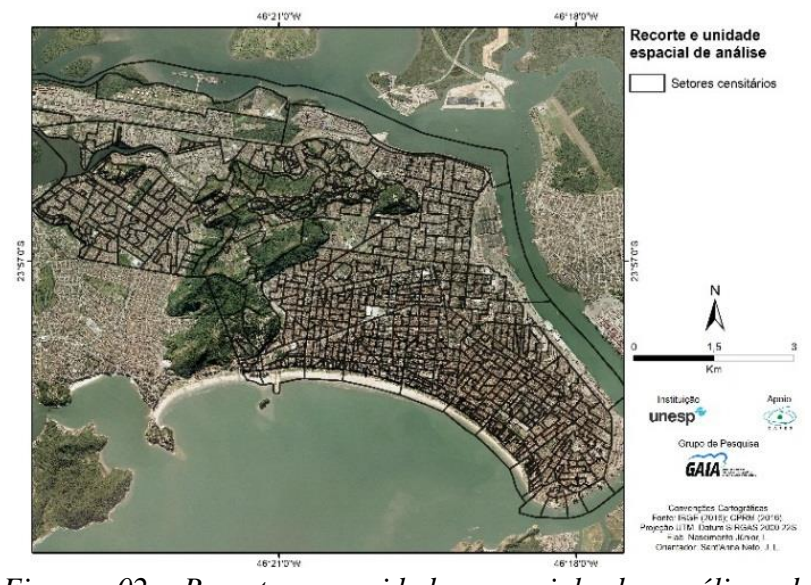

Figura 02. Recorte e unidade espacial de análise da vulnerabilidade para a cidade de Santos - SP.

117 variáveis foram selecionadas e em seguida agrupadas em duas dimensões: a) criticidade - associados à densidade demográfica, condições socioeconômicas, raça/cor, alfabetização, gênero e estrutura etária; e b) capacidade de suporte - que contemplou o perfil dos residentes (responsáveis, idade e gênero), as condições socioeconômicas (bens duráveis e de riqueza material), situação da propriedade (própria, alugada e cedida), condições e tipo de domicílios (casa, vila, apartamento), e infraestrutura (identificação, coleta de lixo, abastecimento, iluminação, rampa, arborização, meio fio, calçada, pavimentação, etc).

Todos os dados foram submetidoas a padronização (transformação em valores porcentuais, utilizando duas variávies ponderadoras - total de domicílios e população residentes por setores censitários). Não foram realizadas transformações separadas para cada dimensão - admitiu-se normalizações no conjunto total dos dados. Esse parâmetro é importante, pois é possível verificar erros de cálculo e de preenchimento, facilitando a correção e o controle de sub/superestimações no produto final.

Depois de normalizados, os valores foram submetidos ao teste de multicolinearidade, admitindo-se omissões das variáveis que não apresentaram correlações dom valores de $r^{2}>0,09$. Segundo Hair Jr. et al. (2005, p.142) os efeitos da multicolinearidade merece um cuidado que é de avaliar quando os pesos relativos dos coeficientes de correlação podem não representar a intensidade, com que determinada variável (independente) é capaz de explicar as demais variáveis (dependentes). Esse processo contemplou as variáveis estatisticamente significativas para representação quantitativa em cada setor censitário.

Os pesos obtidos pela multicolinearidade reduziram as 117 variáveis em um grupo de 77 . Essas por sua vez foram submetidas à decomposição espectral pela técnica da Análise Componente Principal. A contribuição de cada componente foi admitida pelo autovalor acumulado até $75 \%$ da variancia da matriz, cujo o interesse foi ordenar padrões estatísticos para construção do SoVI ${ }^{\circledR}$. Admitiu-se cargas fatoriais significativas para aplicação aquelas entre $-0,5$ a 0,5, conforme Cutter et al (2003). 
Os valores finais foram ajustados para obtenção da cardinalidade das componentes principais positivas $(+)$ e negativas (-), garantindo as cargas que associam aumento e diminuição da vulnerabilidade, respectivamente. Uma vez determinadas as cardinalidades, todos os valores foram submetidos ao cálculo da média aritmética para determinar o escores de indicação numérica da vulnerabilidade, e em seguida os valores foram classificados pela técnica de desvio padrão. Foram considerados em classes de vulnerabilidade os escores agrupados entre muito baixa, baixa, moderada baixa, moderada alta, alta e muito alta.

Todos os cálculos estatisticos foram exercutados no Microsoft Office ${ }^{\circledR}$ com o pacote XLStat ${ }^{\circledR}$. O processamento cartográfico foi desenvolvidos em ambiente de Sistemas de Informações Geográficas - SIG com o software ArcGIS ${ }^{\circledR}$.

\section{RESULTADOS E DISCUSSÃO}

A composição do SoVI ${ }^{\circledR}$ para a cidade de Santos apresentou como resultado final nove componentes principais, com variância explicativa de $75,6 \%$, conforme Tabela 01 .

Tabela 01 - Componentes principais do SoVI aplicado à cidade de Santos. Elaboração: Autores.

\begin{tabular}{clc}
\hline Fator & \multicolumn{1}{c}{ Componentes } & $\begin{array}{c}\text { Percentual } \\
\text { explicativo }\end{array}$ \\
\hline $\mathbf{1}$ & Desigualdade (social, gênero e raça) & 29,98 \\
\hline $\mathbf{2}$ & Moradia e residência & 13,21 \\
\hline $\mathbf{3}$ & Abastecimento & 9,12 \\
\hline $\mathbf{4}$ & Estrutura familiar & 8,37 \\
\hline $\mathbf{5}$ & Rendimento & 4,79 \\
\hline $\mathbf{6}$ & Riqueza material & 4,14 \\
\hline $\mathbf{7}$ & Qualidade ambiental & 3,36 \\
\hline $\mathbf{8}$ & \multirow{2}{*}{ Saneamento } & 3,11 \\
\hline $\mathbf{9}$ & & 2,56 \\
\hline
\end{tabular}

A $1^{\text {a }}$ componente $(26,98 \%$ de percentual explicativo $)$ apresentou como variável dominante (ou de maior carga fatorial) os domicílios sem rendimento mensal nominal per capta, e em seguida as variáveis de alfabetização, gênero, raça, e faixa etária. Implica considerar que, em Santos, a $1^{\circ}$ componente da vulnerabilidade social de Santos é a Desigualdade (social, de gênero e raça). A interpretação sugere que jovens alfabetizadas, portadoras da cor parda e residentes em domicílios improvisados sem rendimento formam as populações mais vulneráveis a desastres naturais, considerando o conjunto de variáveis cujo as cargas fatoriais são maiores que 0,5 .

A $2^{a}$ componente explica $13,21 \%$ da variância da matriz da vulnerabilidade da cidade santista. As variáveis que formam os escores dessa componente sugerem que as populações mais vulneráveis são as aquelas residem em domicílios alugados, com a presença de iluminação pública, pavimentação, identificação, meio fio, calçada, bueiro/boca de lobo e arborização. A interpretação da $2^{\mathrm{a}}$ componente indicar que a vulnerabilidade em Santos seja atribuída ao acesso à terra e moradia urbana de qualidade. Ela então pode ser interpretada a partir dos limites de acesso à Moradia e residência.
Com $9,12 \%$ de variância explicativa, a $3^{\mathrm{a}}$ componente da vulnerabilidade em Santos apresenta como variáveis dominantes domicílios particulares com outras formas de abastecimento de água, que segundo o IBGE (2010), se referem as provenientes de poços, carros-pipa, águas pluviais e fluviais (rios, açudes, lagos e igarapés), e demais fontes diferentes das já descritas. Assim, com base na variável dominante, é possível interpretar que vulnerabilidade em Santosestá atribuída em terceiro lugar às formas de Abastecimento.

Estrutura familiar é a $4^{\mathrm{a}}$ componente principal da vulnerabilidade em Santos. Apresta $8,37 \%$ de variância, pelo domínio dos variáveis domicílios particulares permanentes com mais de 10 moradores, cuja combinação dos scores associa também aspectos de saneamento com exemplificação da variável domicílios particulares permanentes com lixo coletado.

Com variância explicativa de $4,79 \%$ o Rendimento sugere a interpretação da $5^{\text {a }}$ componente da vulnerabilidade em Santos. A detecção é observada a partir da variável dominante que é dos domicílios particulares com rendimento mensal domiciliar de $1 / 8$ a 1 salário mínimo. Na mesma componente, a variância se completa com a variável moradora de domicílios particulares permanentes próprios - com identificação, sugerindo associação entre rendimento e riqueza material.

A Riqueza Material e Qualidade Ambiental são a $6^{\mathrm{a}}$ e $7^{\mathrm{a}}$ componentes, e explicam respectivamente $4,14 \%$ e $3,36 \%$ a vulnerabilidade em Satnos. Elas podem ser interpretadas a partir da frequência de variáveis que sugerem bens materiais adquiridos/privados, referem-se aos moradores de domicílios particulares permanentes próprios (variável dominante da componente), domicílios particulares permanentes com moradia adequada, com moradia cedida e alugada.

A $8^{a}$ e $9^{a}$ componentes, com $3,11 \%$ e $2,56 \%$ variância da matriz, indicam Saneamento com fator de vulnerabilização. As variáveis dominantes são domicílios particulares permanentes com banheiros de uso exclusivo dos moradores ou sanitário e esgotamento sanitário via fossa séptica e domicílios particulares permanentes com banheiros de uso exclusivo dos moradores ou sanitário e esgotamento sanitário via rio, lago ou mar. As variáveis que completam os escores são os domicílios particulares permanentes com outro destino do lixo.

Quando os índices são espacializados, a vulnerabilidade em Santos tende ocorrer de forma bastante regionalizada, sendo mais recorrentes os altos valores de SoVI ${ }^{\circledR}$ nos setores Norte e Noroeste, que compreendem a Zona Portuária, o setor Central e as principais áreas de aglomerados subnormais (Figura 03). Os menores índices de vulnerabilidade estão distribuídos na orla praiana, no setor Sul da cidade. Valores médios, que indicam vulnerabilidade em níveis moderados, ocorrem na região de contato entre a orla e o setor Norte, e grande parte do setor Oeste, conectando com altos índices as áreas de aglomerados subnormais.

A interpretação da vulnerabilidade em Santos sugere certa descontinuidade da relação que existe entre os padrões construtivos das habitações e das moradias com as condições sociais e econômicas das populações. Como a vulnerabilidade é do lugar, é possível inferir que essa espacialidade é um dos resultados de um processo histórico de urbanização, cujas estruturas e conjunturas socioespaciais evidenciam a produção do 
espaço urbano em uma totalização que é produto-produtora de riscos (NASCIMENTO Jr, 2018).

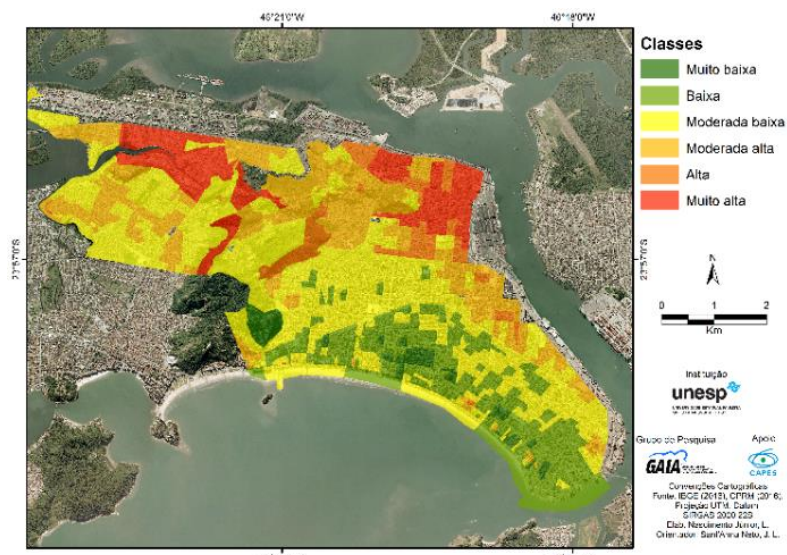

Figura 03 - Mapa de vulnerabilidade social à desastres naturais para a cidade de Santos - SP.

Neste caso, a atuação de diversos agentes sociais na história da cidade definiu processos de urbanização (higienista, verticalizações, segregação, marginalização e favelização) que ao mesmo tempo em que precarizou e segregou o espaço urbano, também definiu áreas naturalmente frágeis e suscetíveis à desastres naturais como lugares de moradia e habitação. Essas fundamentalmente ocupadas por populações pobres e negras. Diferentemente do setores sul onde a infraestrutura é satisfatória, e a redundante rede de infraestrutura e de serviços se dimensiona com verticalização predial e atividades para o setor do turismo. (NASCIMENTO Jr, 2018).

A título de exemplificação, na validação remota do SoVI ${ }^{\circledR}$ é possível comparar o Morro Santa Terezinha (bairro com alto padrão, presença de piscinas, loteamentos extensos) com os menores índices de vulnerabilidade observados. Enquanto, o bairro vizinho - Morro José Menino, apresenta além dos altos índices de vulnerabilidade, a ocupação por loteamentos menores e precarizados (Figura 04).

Destaca-se no por isso que, além dos processos estatísticos de controle da qualidade do índice desenvolvidos durante o processo de elaboração, a validação remota sugere ajustes representativos satisfatórios para análise do mapeamento. Neste sentido, é possível inferir que a vulnerabilidade social à desastres naturais em Santos está organizada na combinação das componentes (por meio dos perfil populacionais vulnerabilizados) e espacialização dos índices (que pode indicar processos de regionalização e setorização de políticas públicas).

De todo modo, o que se interpreta é que a vulnerabilidade social a desastres naturais em Satnos corresponde como subproduto de um Estado que produziu o espaço urbano e: a) destinou áreas ambientalmente frágeis e naturalmente suscetíveis e expostas a eventos perigosos com o locais de habitação e moradia paras as populações empobrecidas e marginalizadas; b) estruturou um processo de urbanização baseado na instalação de infraestruturas e sistemas de engenharia (até certa medida sofisticados) para controle de eventos extremos de base higienista.

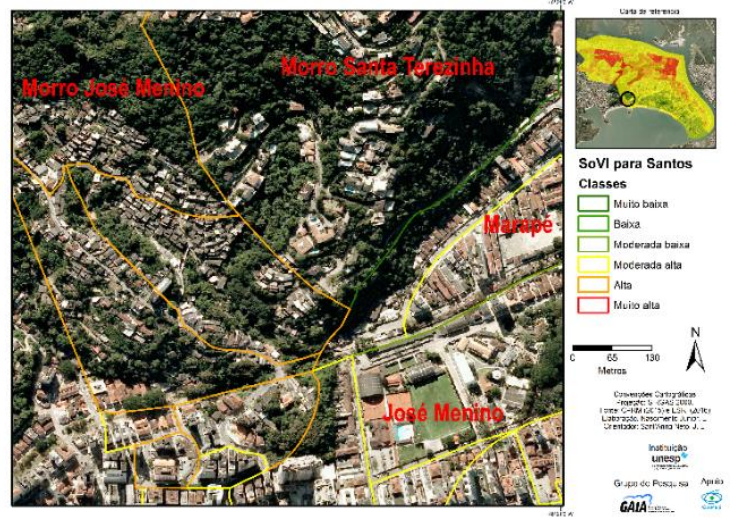

Figura 04 - Validação remota dos valores do SoVI ${ }^{\circledR}$ na cidade de Santos - SP.

O resultado foi a organização de uma segregação socioespacial e racial expressa na produção classista do espaço urbano. Ele se baseia em estrutura fundiária que favorece a concentração da riqueza, coloca limites de acesso à terra e aos bens materiais associados, e segmenta a população em dois setores diferentes da ilha - sendo a primeira para Região Noroeste e na periferia da Zona Portuária destinados à grupos sociais pobres e negros, e a segunda, no Setor Sul da Orla da Praia, aos grupos sociais mais enriquecidos. De um lado o estimulo à valorização imobiliária com infraestrutura eficiente, e do outro, a precarização, a segregação, a periferização e a favelização (NASCIMENTO Jr, 2018).

É possível considerar assim que, os agentes produtores de espaço urbano santista tem utilizado a cidade para diferenciar, desigualizar, segregar e vulnerabilizar a população. Trata-se de uma vulnerabilidade historicamente construída pelo Estado, e que atualmente, integrada aos agentes sociais do mercado imobiliário, tende a ser mantida para a continuidade da segregação socioespacial e da vulnerabilizando populações na cidade.

Esse argumento contempla todos os programas habitacionais e de moradia, e os instrumentos do planejamento urbano que garantiram, de forma bastante eficiente e cada um em sua particularidade, o acesso à terra como ferramenta de controle e manutenção da concentração da riqueza, combinando-as com limites à garantia de áreas com serviços básicos e infraestruturas sofisiticadas para proteção desigual e controle seletivo de desastres naturais.

\section{CONSIDERAÇÕES FINAIS}

Desde que passe por processos metodológicos coerentes, a aplicação do SoVI ${ }^{\circledR}$ pode ser uma opção viável e eficaz para análise da vulnerabilidade em áreas urbanas. Neste trabalho preferiu-se optar pela integração de variáveis em um banco de dados único - Censo Demográfico, utilizando-se os setores censitários. Mas os resultados não limitam possibilidades de comparação e aplicação com outras áreas urbanas, bem como a união com outros bancos de dados. Isso inclusive deve fomentar formas de avaliação dos modelos mais recorrentes de análise da 
vulnerabilidade incorporando ainda mais complexidade e potencial representatico do índice.

Do mesmo modo, a explicação dos produtos finais também deve estar ancorada em suportes conceituais e teóricos que explicam a organização e estruturação da vulnerabilidade no lugar. O índice assim torna-se uma estratégia de debate e um instrumento político, na medida em que, auxilia o entendimento dos processos de vulnerabilização e pode servir para integrar a luta por justiça e direitos sociais.

No caso de Santos, a vulnerabilidade explicita como a produção histórica da desigualdade e da segregação socioespacial garantiu ou bloqueou direitos sociais frente aos riscos. O resultado é a organização das jovens, alfabetizadas, portadoras da cor parda e residentes em domicílios improvisados sem rendimento, como o grupo populacional mais vulnerável. E os setores norte, que compreende a zona portuária, o centro e as áreas de aglomerados subnormais os lugares mais vulnerabilizados, e potencialmente expostoss a qualquer desastre.

Os resultados apresentam parâmetros de explicação de como a aquisição da terra urbanizada evidencia os níveis de vulnerabilidade em Santos, e estruturam a produção do espaço urbano santista como uma cidade segmentada, segregada e desigual. Essa situação pode ser admitida como uma generalização da vulnerabilidade em outras áreas urbanas, não exclusiva às brasileiras e às do mundo tropical colonizado, mas representativa destas.

\section{REFERÊNCIAS}

ACSELRAD, H. Vulnerabilidade ambiental, processos e relações. In: II Encontro Nacional de Produtores e Usuários de Informações sociais, econômicas e territoriais. Rio de Janeiro. Comunicação. Rio de janeiro: Instituto Brasileiro de Geografia e Estatística (IBGE), 2006.

ALMEIDA, L. Q. Riscos ambientais e vulnerabilidades nas cidades brasileiras: conceitos, metodologias e aplicações. Cultura Acadêmica. São Paulo. 2012.

ANDERSON, P. Vulnerability to disaster and sustainable development: a general framework fo assessing vulnerability. In: MUNASINGHE, M, CLARKE, C. Disaster prevention for sustainable development: econômica and policy issues. Washingtton: IDNDR. The world bank, 1995, p. $41-59$.

ARMOND, N. Dinâmica climática, excepcionalidades e vulnerabilidade: contribuições para uma classificação geográfica do clima do estado do Rio de Janeiro. Tese (Doutorado em Geografia) Universidade Estadual Paulista. Presidente Prudente. 2018.

BIRKMANN, J. Measuring Vulnerability to Natural Hazards: Towards disaster resilient societies. Toquio: United Nations University Press. 2006.

CUNHA, L. Vulnerabilidade: a face menos visível do estudo dos riscos naturais. In LOURENÇO, L; MATEUS, M. Riscos naturais, antrópicos e mistos. Homenagem ao Professor Fernando Rebelo. Coimbra, 2013, p. 153-165.
CUTTER, S. L. A ciência da vulnerabilidade: modelos, métodos e indicadores. Revista Crítica de Ciências Sociais, v. 93, n. 1, p. 59-70, jun. 2011.

CUTTER, S. L. The vulnerability of Science and the Science of vulnerability. Annals of the Associaton of American Geographers, v. 93, n 1. 2003.

CUTTER, S. L; BORUFF, J.; SHIRLEY, W. Social vulnerability to environmental hazards. Social Science Quarterly, v. 84, n. 2, p. 242-261, 2003.

DAUPHINÉ, A. Risques et catástrofes: observer, spatialiser, comprendre, gérer. Paris. Armand Colin. 2005.

DESCHAMPS, M. V. Estudo sobre a vulnerabilidade socioambiental na Região Metropolitana de Curitiba. Cadernos Metrópole, n. 19, $1^{\circ}$ sem, 2008, p. 191219.

DUTRA, A. S. Problematizando o conceito de risco. O Social em Questão, v. 1, n. 33, 2015, p. $177-192$.

HUMMELL, B; M. L; CUTTER, S. L.; EMRICH, C. T. Social vulnerability to natural hazards in Brazil. International Journal of Disaster Risk Science, v. 7, n. 2, p. 111-122, 2016.

IBGE. Instituo Brasileiro de Geografia e Estatística. Censo demográfico - Resultado do universo. Rio de Janeiro. 2010.

LEONE, F; VINET, F. La vulnerabilité, un concept fondamental au coeur des méthodes d'évaluation des risques naturels. In : LEONE, F.; VINET, F. La vulnerabilité des sociétés et des territoires face aux menaces naturelles: analyses géographiques. Montpellier : Université Paul Valery, Collection Géorisques n. 1, 2006,

LIEBER, R. R; ROMANO-LIEBER, N. S. O conceito de risco: Janus reinventado. In: MINAYO, Maria Cecília de Souza; MIRANDA, Ary Carvalho de (orgs.). Saúde e ambiente sustentável: estreitando nós. Rio de Janeiro: Editora FIOCRUZ, 2002, p. 69-111.

MELLO, G. H. Expansão e estrutura urbana de Santos (SP): aspectos da periferização, da deterioração, da intervenção urbana, da verticalização e da sociabilidade. Tese de Doutorado. Universidade de São Paulo. 2007.

MENDES, J. M. TAVARES, A. O; CUNHA, L; FREIRIA, S. A vulnerabilidade social aos perigos naturais e tecnológicos em Portugal. Revista Crítica de Ciências Sociais, n. 93, p. 95-128, 2011.

NASCIMENTO Jr, L. Clima urbano, risco e vulnerabilidade em cidades costeiras do mundo tropical: estudo comparado entre Santos (Brasil), Maputo (Moçambique) e Brisbane (Austrália). 2018. Tese (Doutorado em Geografia) Universidade Estadual Paulista. Presidente Prudente. 2018.

RAMPAZZO, Camila Riboli. Clima urbano, risco climático e vulnerabilidade socioespacial mediados pela produção do espaço urbano em cidades paulistas (São Carlos, Marília e 
Presidente Prudente). Tese (Doutorado em Geografia) Universidade Estadual Paulista. Presidente Prudente. 2019.

ROSS, J. MOROZ, I. C. Mapa geomorfológico do estado de São Paulo. Revista do Departamento de Geografia, v. 10, p. 4158, 2011.

SILVEIRA, R. D. Risco climático, vulnerabilidade socioespacial e eventos climáticos extremos relacionados ao calor e ao frio no estado do Rio Grande do Sul-Brasil. Tese (doutorado em Geografia). Universidade Estadual Paulista. Presidente Prudente. 2014.

SMITH, K. Environmental hazards: assessing risk and reducing disaster. 3a. ed. London: Routledge, 2001.

TEOBALDO NETO, A. A geografia do risco $e$ da vulnerabilidade ao calor em espaços urbanos da zona tropical: o caso Cuiabá/MT. Tese (Doutorado em Geografia) Universidade Estadual Paulista. Presidente Prudente. 2019.

VEYRET, Y. Os riscos. O homem como agressor e vítima do meio ambiente. São Paulo. Contexto, 2007.

WHITE, G. F; KATES, R. W; BURTON, I. Knowing better and losing even more: the use of knowledge in hazards management. Environmental hazards. v. 3, n. 3-4, set./dez., p. 81-92, 2001.

\section{AGRADECIMENTOS}

Os autores agradecem à Coordenação de Aperfeiçoamento de Pessoal de Nível Superior, pelo apoio financeiro durante o período de 2014-2017. Ao Grupo de Pesquisa GAIA - Interações na superfície, água e atmosfera da UNESP/Presidente Prudente pela infraestrutura e ambiente agradável e amigável. E à comissão organizadora e científica do XVIII Simpósio Brasileiro de Geografia Física Aplicada pela indicação para publicação do artigo no presente periódico.

Recebido em: 19/11/2019

Aceito para publicação em: 10/06/2020 